\title{
Validation of Glomerular Filtration Rate Measurement with Blood Sampling from the Injection Site
}

\author{
Bethelhem W. Mekonnen, James M. Warwick, and Jennifer L. Holness \\ Nuclear Medicine Division, Department of Medical Imaging and Clinical Oncology, Stellenbosch University, Cape Town, South \\ Africa
}

\begin{abstract}
Measurement of glomerular filtration rate (GFR) from the plasma clearance of a radionuclide-labeled tracer is reliable and accurate. However, to avoid contamination of the blood samples with radioactivity remaining at the injection site, venepuncture at 2 or more sites is required: one for tracer administration and the others for blood sampling. This requirement is uncomfortable for patients, particularly when venous access is difficult. The objective of this study was to validate the use of a single site of venous access in combination with injection site imaging, for GFR measurement. Methods: Twenty-two adults ( $\geq 18 \mathrm{y}$ ) who were referred for GFR determination were included prospectively. GFR was measured from the plasma clearance of $99 \mathrm{mTc}$-diethylenetriaminepentaacetic acid according to international guidelines. After administration of the tracer through an intravenous cannula, a 60-s static image of the injection site was acquired. A second intravenous cannula was inserted into the contralateral arm. Venous blood samples were collected at 2,3 , and $4 \mathrm{~h}$ after administration of the radiotracer from both the injection site (experimental) and the contralateral arm (conventional). GFR was calculated using slope-intercept and single-sample methods. The median conventional and experimental plasma counts (decay- and background-corrected) were compared for the 2-, 3-, and 4-h venous samples. Conventional and experimental GFRs were then compared, with a more than $10 \%$ difference between conventional and experimental GFRs being regarded as significant. Results: Four individuals had visible residual activity at the injection site. The median $2-h$ counts differed significantly between the conventional and experimental sampling sites $(P=0.007)$, whereas no significant difference was found at 3 or $4 \mathrm{~h}$. When there was a clear injection site image, the difference between the experimental and conventional GFRs was more than $10 \%$ in 1 case for single-sample GFR but less than $8 \%$ in all cases for slope-intercept GFR. Conclusion: In cases with clear injection site images, slope-intercept GFR calculated after injection site blood sampling showed no clinically significant difference from conventional contralateral-arm sampling.
\end{abstract}

Key Words: glomerular filtration rate; blood specimen collection; cannula; adult; radioactivity

J Nucl Med Technol 2019; 47:305-308

DOI: 10.2967/jnmt.119.229153

\footnotetext{
Received Mar. 28, 2019; revision accepted May 21, 2019.

For correspondence or reprints contact: Bethelhem W. Mekonnen, Department of Medical Imaging and Clinical Oncology, Nuclear Medicine

Division, P.O. Box 241, Cape Town, 8000, South Africa.

E-mail: bety192112@gmail.com

Published online Jun. 10, 2019.

COPYRIGHT (C) 2019 by the Society of Nuclear Medicine and Molecular Imaging.
}

$\mathbf{G}$ lomerular filtration rate (GFR) is a standard measure of renal function (1). GFR represents the plasma volume presented to the nephrons per unit time during urine formation. It is usually measured in milliliters per minute (1). Radionuclide-based techniques allow for rapid and reliable measurement of GFR from plasma samples taken after intravenous administration of a bolus of radionuclide-labeled tracer (2). GFR is frequently measured from the plasma clear-

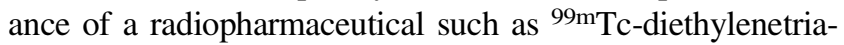
minepentaacetic acid or ${ }^{51} \mathrm{Cr}$-ethylenediaminetetraacetic acid. Although the plasma clearance of ${ }^{51} \mathrm{Cr}$-ethylenediaminetetraacetic acid is often considered the standard measure of GFR, particularly in Europe, ${ }^{99} \mathrm{~m}$ Tc-diethylenetriaminepentaacetic acid gives similar results with added advantages of being cheaper and more widely available, having higher counting efficiency, and allowing for simultaneous imaging $(1,3)$.

Radionuclide-based GFR determination in general is relatively time-consuming, labor-intensive, and uncomfortable for the patient. There is thus a need for the technique to be simplified without compromising the accuracy of the result. Current international guidelines for the measurement of GFR state that the tracer should not be injected through the same line as that used for blood sampling (4). This guideline is to eliminate the risk of contamination of the blood samples by residual radioactivity in the line (2). Consequently, venous access must be obtained from at least 2 sites, usually in each arm. Frequently, large-bore intravenous catheters, needles, or butterfly catheters used for this purpose are uncomfortable for patients and may be distressing for children. In addition, venous access may be difficult in certain individuals (e.g., young children, the elderly, obese patients, or patients receiving chemotherapy), making finding 2 sites of access more challenging. Difficult access also increases the risk of hemolysis of the samples, which may invalidate the measurements (1). Some centers prefer to place an intravenous cannula with a valve, allowing both tracer injection and repeated blood sampling with only 1 venepuncture (5). However, these may not be routinely available, particularly in resource-limited settings.

Sample contamination may, however, be insignificant if $\gamma$-camera imaging, performed immediately after tracer administration, detects no residual activity in the intravenous 
or butterfly catheter. If this is indeed the case, a strong argument can be made to support the use of a single intravenous or butterfly catheter for both administration of activity and blood sampling in combination with imaging. This change would simplify the procedure and reduce patient discomfort. A rigorous validation of this methodology is likely to contribute to the development of new guidelines; however, there is limited published research that addresses this issue. Therefore, the aim of this study was to validate the use of a single site of venous access in combination with injection site imaging, for GFR measurement.

\section{MATERIALS AND METHODS}

The study was approved by the Health Research Ethics Committee of Stellenbosch University (protocol S17/10/191), and all subjects signed an informed consent form. Adults ( $\geq 18 \mathrm{y}$ ) referred for GFR determination at Tygerberg Hospital, Cape Town, South Africa, over a 6-mo period (April to September 2018) were invited to participate. Subjects with difficult venous access were excluded.

The GFR studies were performed following departmental protocol, which is based on a 2004 guideline of the British Nuclear Medicine Society (1). A median dose of $45 \mathrm{MBq}(1.2$ $\mathrm{mCi}$ ) (range, 36-49 MBq [0.97-1.3 mCi]) of ${ }^{99 m} \mathrm{Tc}$-diethylenetriaminepentaacetic acid (Renatek; NTP Radioisotopes Ltd.) was administered as a bolus through 20- or 22-gauge intravenous cannulae. In all patients, a 60-s static image of the injection site was acquired between 5 and 93 min after injection with the intravenous cannula in situ using 1 of 3 available $\gamma$-cameras: a Siemens Symbia (low-energy all-purpose collimator), a GE Healthcare Infinia, or a GE Healthcare Hawkeye (low-energy high-resolution collimators). If there was visible activity at the injection site on this image, its quantity was calculated and expressed as a percentage of administered activity, based on previously determined camera sensitivities.

A second 18- or 20-gauge intravenous cannula was inserted into the contralateral arm. At 2, 3, and $4 \mathrm{~h}$ after radiotracer administration, venous blood samples $(\sim 10 \mathrm{~mL})$ were collected simultaneously from this contralateral arm (according to normal practice) and from the injection site. For practical reasons, the blood samples from the contralateral arm and the injection site were collected by different people, a technologist and the principal investigator, respectively. A small volume $(\sim 1 \mathrm{~mL})$ of heparinsaline was injected into both intravenous cannulae after blood sampling to maintain cannula patency, and before the next sample was taken, at least $3 \mathrm{~mL}$ of blood were first drawn and discarded. Blood samples from the contralateral arm were denoted conventional, and those from the injection site were denoted experimental.

The conventional and experimental samples were handled identically. Blood samples were centrifuged at $1,000 \mathrm{~g}$ for $10 \mathrm{~min}$, duplicate 1-mL plasma and standard samples were pipetted into counting tubes, and all samples were counted simultaneously with a multichannel well counter (Videogamma 4880; l'acn Scientific Laboratories) following departmental protocol. The same standard samples were used for conventional and experimental GFR calculations. Body surface area in square meters was calculated using the Haycock formula, with weight in kilograms and height in centimeters (weight ${ }^{0.5378} \times$ height $\left.^{0.3964} \times 0.024265\right)(6)$.

GFR was calculated using slope-intercept (7) and single-sample (8) methods. Slope-intercept GFR was corrected using the mean
Bröchner-Mortensen equation (1,9,10). Routine quality control checks were performed for both methods.

In cases with clear injection site images, the average plasma counts (decay- and background-corrected) were calculated for the 2-, 3-, and 4-h samples from both conventional and experimental sites. A Wilcoxon test was used to compare the median counts. The Shapiro-Wilk test was used to test for normality.

Slope-intercept GFR was calculated using all 3 plasma samples (2, 3, and $4 \mathrm{~h})$, and the 2-h sample was used for single-sample GFR calculation. To determine agreement between conventional and experimental slope-intercept GFR and between conventional and experimental single-sample GFR, Bland-Altman analyses were performed and the differences were calculated. The proportion of cases with differences of more than $10 \%$ was calculated. A $10 \%$ threshold was selected because it is the coefficient of variation for repeat GFR measurements (11). Statistical analysis was performed using MedCalc (version 18.11.3; MedCalc Software bvba) for Microsoft Windows.

\section{RESULTS}

Twenty-two subjects were included (15 women and 7 men; median age, 53 y; age range, 26-67 y). Twenty-one participants were known to have cancer and were referred for prechemotherapy GFR determination. One participant was a healthy volunteer. The absolute GFR ranged between 68 and $122 \mathrm{~mL} /$ min (median, $79.5 \mathrm{~mL} / \mathrm{min}$ ), and the GFR corrected for body surface area ranged between 60 and $118 \mathrm{~mL} / \mathrm{min} / 1.73 \mathrm{~m}^{2}$ (median, $89 \mathrm{~mL} / \mathrm{min} / 1.73 \mathrm{~m}^{2}$ ). Of the 22 participants, 4 had visible activity at the injection site, ranging between $0.09 \%$ and $0.3 \%$ of the administered dose.

The median 2-h counts significantly differed between the conventional and experimental sampling sites $(P=0.007)$, whereas no significant difference was found at 3 or $4 \mathrm{~h}$. The median difference between experimental and conventional counts, expressed as a percentage of conventional counts, was $4.4 \%$ at $2 \mathrm{~h}, 0.3 \%$ at $3 \mathrm{~h}$, and $-1.9 \%$ at $4 \mathrm{~h}$.

The median differences between the experimental and conventional GFRs were $-1.2 \%$ with the slope-intercept method and $-2.9 \%$ with the single-sample method (Table 1). For single-sample GFR, the difference between the experimental and conventional measurements was more than $10 \%$ in 1 case, whereas for slope-intercept GFR the difference was less than $8 \%$ in all cases. This finding is illustrated by the Bland-Altman analyses (Fig. 1). In 3 of the 4 cases with visible residual activity, the experimental slope-intercept and single-sample GFRs were higher than the conventional GFRs by $20-80 \mathrm{~mL} / \mathrm{min} / 1.73 \mathrm{~m}^{2}$, well beyond the $95 \%$

TABLE 1

Median Difference Between Experimental and Conventional GFR in Cases with Clear Injection Site $(n=18)$

\begin{tabular}{ccc}
\hline Method & $\mathrm{mL} / \mathrm{min} / 1.73 \mathrm{~m}^{2}$ & Percentage \\
\hline $\begin{array}{c}\text { Slope } \\
\text { intercept }\end{array}$ & -1.3 (range, -6.9 to 6.0$)$ & -1.2 (range, -7.5 to 6.9$)$ \\
$\begin{array}{c}\text { Single } \\
\text { sample }\end{array}$ & -2.6 (range, -10.0 to 2.9$)$ & -2.9 (range, -10.9 to 3.3 ) \\
\hline
\end{tabular}




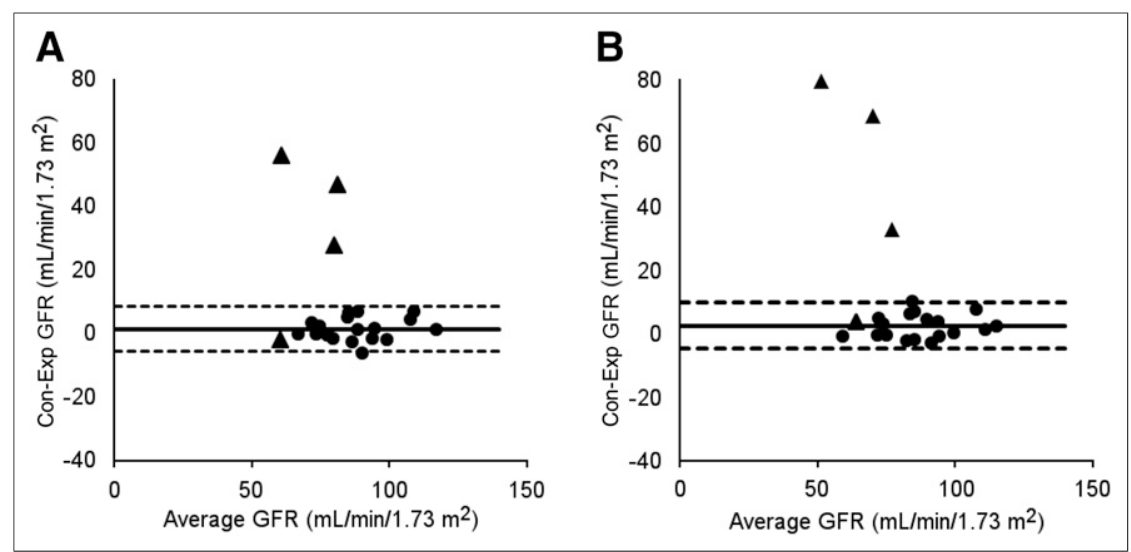

FIGURE 1. Bland-Altman plots of conventional vs. experimental GFR in cases with clear injection site, using slope-intercept (A) and single-sample (B) methods. Solid lines represent mean differences and dashed lines upper and lower $95 \%$ limits of agreement. Triangles represent 4 cases with visible activity at injection site and are plotted for purposes of comparison. Con-Exp = conventional minus experimental GFR.

sample GFR, 1 case differed by $12.7 \%$. It can therefore be argued, from a clinical point of view, that it is acceptable to use injection site blood sampling to determine GFR, at least in patients with challenges to venous access.

Our findings are in line with a previous report by Brändström et al. (14) in which no difference in GFR was found between contralateral-arm and injection site blood sampling. The authors did, however, stress that the injection site venous catheter was flushed with at least $30 \mathrm{~mL}$ of saline after radiotracer injection.

In another study, by Gawthorpe et al. (15), it was concluded that a singlelumen central venous catheter should not be used for tracer injection and blood sampling because the GFR re-

limits of agreement derived from cases without visible activity (Fig. 1).

\section{DISCUSSION}

GFR is the best index of kidney function in health and disease, and accurate values are required for optimal decision making (12). Evaluation of GFR using a bolus injection of a radionuclide tracer and measurement of its plasma clearance has become a widely used method to assess kidney function (13). However, the investigation is time-consuming, laborintensive, and uncomfortable for the patient.

In this study, we compared the conventional technique of contralateral-arm blood sampling to an experimental method with blood samples taken through the injection site cannula, after imaging the injection site to exclude the presence of scintigraphically detectable residual injected activity. The median counts at $2 \mathrm{~h}$ were significantly higher at the experimental sampling site than at the conventional site $(P=$ $0.007)$, whereas no significant difference was found at 3 or $4 \mathrm{~h}$. This finding is most likely due to contamination of the 2-h samples by higher occult residual activity, which is likely to have decreased in later samples as a consequence of previous sampling.

As expected, experimental GFR was extremely inaccurate in 3 of the 4 patients with visible activity at the injection site. This finding confirms the need for injection site imaging (or counting over the injection site) to detect residual activity at the injection site, thus precluding the performance of injection site blood sampling. In the 18 cases with clear injection site images, the percentage differences between the conventional and experimental sites were all less than $8 \%$ for slope-intercept GFR (range, $-6.9 \%$ to $7.5 \%$ ). However, for single-sample GFR, in 1 case the difference was more than $10 \%$, with the percentage difference ranging from $-3.1 \%$ to $12.7 \%$. Using a $10 \%$ threshold, there was no clinically significant difference in slope-intercept GFR between the conventional and experimental sites. For single- sult was significantly affected by contamination. In our study, although sample contamination affected the 2-h sample counts, the effect on GFR measurement was not clinically significant, especially if slope-intercept GFR was used. It can be speculated that the addition of a saline flush or discarding of the first blood sample may further reduce the impact of occult activity at the injection site.

This study had a few limitations. The methodology cannot be applied to ${ }^{51} \mathrm{Cr}$-ethylenediaminetetraacetic acid GFR because imaging of the injection site is not possible. A comparable method of measuring residual activity using a handheld monitor would be required. Detection of residual activity at the injection site on an image is related to the sensitivity of the individual camera. Had the department's most sensitive camera been used with an all-purpose collimator in all cases, it is possible that residual activity would have been identified in additional cases. However, it can be argued that use of various cameras reflects clinical practice, in which it is likely that injection site imaging would be allocated to whatever machine is available and that this machine may not be the most sensitive. The number of participants with visible activity at the injection site was small; however, most of these had very inaccurate GFR results. In addition, it is possible that further flushing of the injection site cannula, or obtaining blood samples through direct intravenous access, would have improved the results. These are issues for possible further studies.

\section{CONCLUSION}

In cases with clear injection site images, slope-intercept GFR calculated after injection site blood sampling was not clinically significantly different from that obtained using conventional contralateral-arm sampling. Therefore, single venous access for both blood sampling and radiopharmaceutical injection, combined with injection site imaging, can be used at least in a clinical setting where patients have difficult venous access. 


\section{DISCLOSURE}

Jennifer Holness received funding from the South African Medical Research Council under the Division of Research Capacity Development under the Clinician Researcher Development PhD Scholarship Programme. No other potential conflict of interest relevant to this article was reported.

\section{REFERENCES}

1. Fleming JS, Zivanovic MA, Blake GM, Burniston M, Cosgriff PS. Guidelines for the measurement of glomerular filtration rate using plasma sampling. Nucl Med Commun. 2004;25:759-769.

2. Murray AW, Barnfield MC, Waller ML, et al. Assessment of glomerular filtration rate measurement with plasma sampling: a technical review. J Nucl Med Technol. 2013;41:67-75.

3. Holness J, Fleming J, Warwick J. Measuring GFR using the plasma clearance of 99mTc-DTPA [reply]. Am J Kidney Dis. 2015;65:806.

4. Burniston M. Clinical Guideline for the Measurement of Glomerular Filtration Rate (GFR) using Plasma Sampling. Nottingham, U.K.: British Nuclear Medicine Society; 2018.

5. Piepsz A, Colarinha P, Gordon I, et al. Guidelines for glomerular filtration rate determination in children. Eur J Nucl Med. 2001;28:BP31-BP36.
6. Haycock GB, Schwartz GJ, Wisotsky DH. Geometric method for measuring body surface area: a height-weight formula validated in infants, children, and adults. J Pediatr. 1978;93:62-66.

7. Chantler C, Garnett ES, Parsons V, Veall N. Glomerular filtration rate measurement in man by the single injection methods using ${ }^{51}$ Cr-EDTA. Clin Sci. 1969;37:169-180.

8. Fleming JS, Persaud L, Zivanovic MA. A general equation for estimating glomerular filtration rate from a single plasma sample. Nucl Med Commun. 2005;26: 743-748.

9. Bröchner-Mortensen J. A simple method for the determination of glomerular filtration rate. Scand J Clin Lab Invest. 1972;30:271-274.

10. Bröchner-Mortensen J, Haahr J, Christoffersen J. A simple method for accurate assessment of the glomerular filtration rate in children. Scand J Clin Lab Invest. 1974;33:140-143.

11. Blake GM, Roe D, Lazarus CR. Long-term precision of glomerular filtration rate measurements using ${ }^{51}$ Cr-EDTA plasma clearance. Nucl Med Commun. 1997;18:776-784.

12. Stevens LA, Levey AS. Measured GFR as a confirmatory test for estimated GFR. J Am Soc Nephrol. 2009;20:2305-2313.

13. Blake GM, Barnfield MC, Burniston MT, Fleming JS, Cosgriff PS, Siddique M. Correction of the slope-intercept method for the measurement of glomerular filtration rate. Nucl Med Commun. 2014;35:1277-1283.

14. Brändström E, Grzegorezyk A, Jacobsson L, Friberg P, Lindahl A, Mattias A. GFR measurement with iohexol and ${ }^{51} \mathrm{Cr}$-EDTA: a comparison of the two favoured GFR markers in Europe. Nephrol Dial Transplant. 1998;13:1176-1182.

15. Gawthorpe BL, Barnfield MC, Burniston MT. Can oncology patients' central venous catheters be used for isotope assessment of glomerular filtration rate? An in-vitro study. Nucl Med Commun. 2007;28:415-421. 